\title{
Signal Processing with Silicon-Organic Hybrid Waveguides
}

\author{
J. Leuthold ${ }^{1}$, C. Koos ${ }^{1}$, W. Freude ${ }^{1}$, T. Vallaitis ${ }^{1}$, L. Alloatti ${ }^{1}$, D. Korn ${ }^{1}$, P. Dumon ${ }^{2}$, W. Bogaerts ${ }^{2}$, R. Baets ${ }^{2}$, \\ I. Biaggio ${ }^{3}$, F. Diederich ${ }^{4}$, \\ ${ }^{1}$ Institute of Photonics and Quantum Electronics, University of Karlsruhe, 76131 Karlsruhe, Germany \\ ${ }^{2}$ Photonics Research Group, Ghent University-IMEC, B-9000 Gent, Belgium \\ ${ }^{3}$ Department of Physics, Lehigh University, Bethlehem, PA 18015, USA \\ ${ }^{4}$ Laboratorium für Organische Chemie, ETH Zürich, Hönggerberg, HCI, CH-8093 Zürich, Switzerland \\ Author e-mail addresses at: www.ipq.kit.edu
}

\begin{abstract}
The silicon-organic hybrid (SOH) platform is reviewed. The $\mathrm{SOH}$ approach is a promising CMOS compatible photonic platform enabling ultrafast nonlinear signal processing in compact silicon photonic devices.

(C)2010 Optical Society of America

OCIS codes: (130.4310) Nonlinear; (130.2790) Guided Waves; (160.4330) Nonlinear optical materials; (190.3270) Kerr effect;

(190.4710) Optical nonlinearities in organic materials; (190.5970) Semiconductor nonlinear optics.
\end{abstract}

\section{Introduction}

Silicon photonics holds promise for convergence of photonic and electronic integrated circuit on one CMOScompatible platform. It has become a likely path towards cost-effective mass-producible, ultra-compact and power efficient multi-functional optoelectronic integrated circuits [1]-[4]. And while the field has made tremendous progress in recent years, electrical and optical modulation is still an issue. As a matter of fact, electrical and optical signal processing suffer from two-photon absorption (TPA) and free carrier absorption (FCA) related speed limitations, which require special measures to keep such speed limitations within limits.

In this paper, we review the silicon-organic hybrid ( $\mathrm{SOH})$ platform [5]. The $\mathrm{SOH}$ platform combines the advantages of silicon with the ultra-fast performance of organic Kerr $\left(\chi^{(3)}\right)$ and electro-optic $\left(\chi^{(2)}\right)$ nonlinear materials. In the $\mathrm{SOH}$ approach the silicon wire guides the optical modes but the necessary nonlinearity for switching is provided by an organic cladding. By a proper choice of the organic material optimum material parameters for the respective application can be chosen. This approach has recently led to demonstrations of $120 \mathrm{Gbit} / \mathrm{s}$ to $10 \mathrm{Gbit} / \mathrm{s}$ [6], $170 \mathrm{Gbit} / \mathrm{s}$ to $43 \mathrm{Gbit} / \mathrm{s}$ TDM demultiplexing [7], and 40 and $56 \mathrm{Gbit} / \mathrm{s}$ RZ and DQPSK wavelength conversion [8]-[10]. More recently, electro-optic switching has been predicted [14]-[17] and confirmed by experiments [18][19]. The electrooptic $\mathrm{SOH}$ approach however is not in the focus of this paper.

\section{The Silicon Organic Hybrid (SOH) Platform}

In the $\mathrm{SOH}$ approach, all active and passive waveguides, couplers and filters, are fabricated as silicon waveguides, see Fig. 1. Nonlinear optical functionality, however, is due to an organic cladding material.

(a)

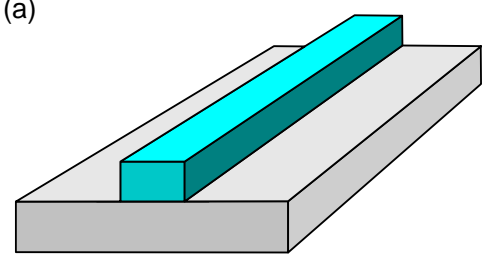

(b)
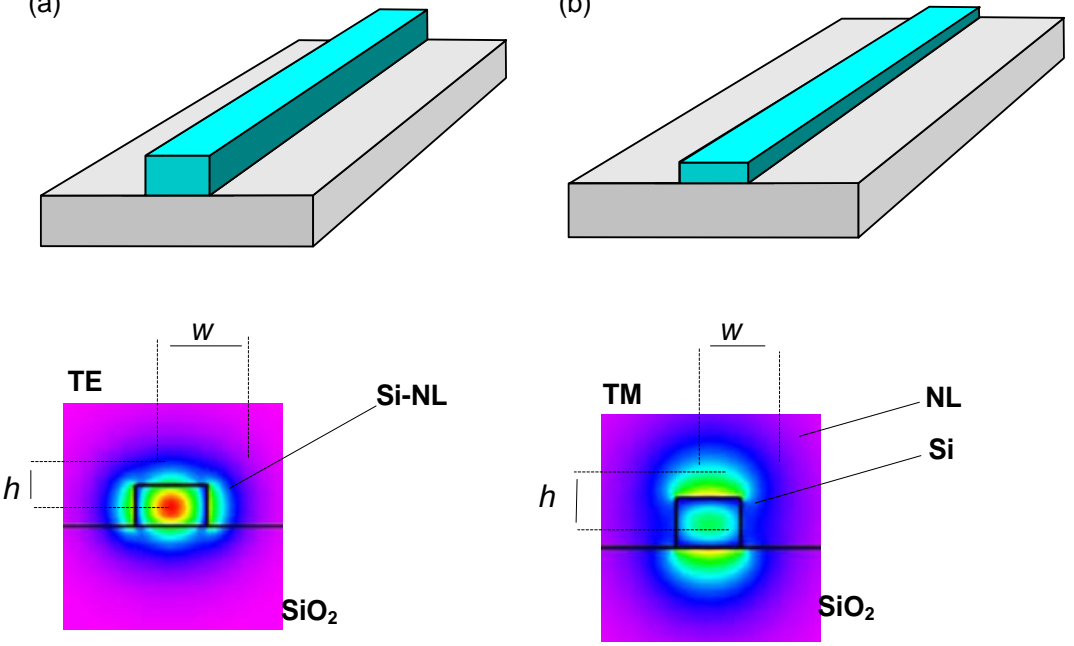

Si-NL (c)
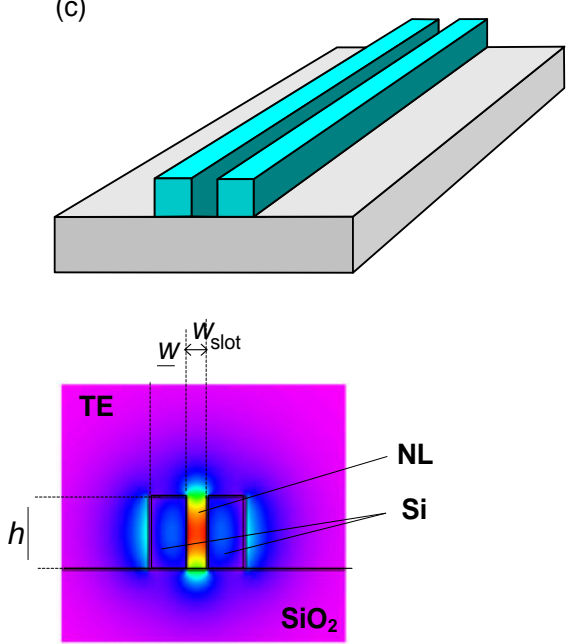

Fig. 1 Waveguide structures and electric field amplitudes of three silicon-wire structures. (a) The conventional silicon strip waveguide providing core nonlinearity from silicon for high-power TE signals, (b) Cover nonlinear waveguide in form of a strip waveguide where an organic cladding provides large nonlinearity to a TM signal, and (c) cover nonlinearity in a slot waveguide where an organic material provides strong nonlinearity to a TE-polarized signal. 


\section{IWC1.pdf}

In the more conventional approach depicted in Fig. 1(a), the dimensions of a silicon strip-waveguide have been chosen to provide a maximum confinement of the optical mode. This way the silicon nonlinearity with a nonlinear index coefficient $n_{2}=6 \times 10^{-18} \mathrm{~m}^{2} / \mathrm{W}$ may be exploited. In the structure of Fig. 1(b) the silicon waveguide provides guiding but is designed to push the optical mode as far as possible into the cladding layer above the waveguide. As a nonlinear material we have chosen the organic molecule DDMEBT with a nonlinear coefficient $n_{2}=2 \times 10^{-17} \mathrm{~m}^{2} / \mathrm{W}$ [11]. A further improvement is obtained with the slot-waveguide structure from Fig. 1(c). Here the optical mode is mostly confined to the narrow slot. The field enhancement across the slot is maximum [11].

Next we compare the nonlinearities of the three waveguide structures depicted in Fig. 2. An in-depth discussion may be found in Ref. [12] and [13]. The strength of the Kerr nonlinearity is best described by the nonlinearity coefficient $\gamma$. The nonlinearity coefficient $\gamma$ can be maximized by using materials with a strong nonlinear index coefficient $n_{2}$ and the smallest possible third-order nonlinear effective interaction area. The nonlinearity coefficient $\gamma$ has been plotted for the three structures in Fig. 2. At first sight it looks as if structure (a) with core nonlinearity has the highest nonlinearity. Yet, the nonlinearity coefficient does not tell all. The efficiency - and even worse the ultrafast short time constants might be impeded by TPA (two-photon absorption). In the presence of large TPA free carriers are generated in the conduction and valence band. These carriers in turn lead to plasma effect related free carrier refractive index and absorption changes. As the free carrier lifetimes are long (up to several $100 \mathrm{~ns}$ ) the overall speed of such structures might be at risk. The bottom part of Fig. 2 shows the evolution of the pump light-induced phase change in the respective three structures in pump-probe experiments While the core nonlinear structure shows a large Kerr-effect it also shows a TPA-induced large plasma effect-related tail with a large time constant. The slot nonlinear structure on the other hand has both a large Kerr effect and no visible plasma effect-related tail. In literature, a figure of merit (FOM) is often used to compare the magnitude of the Kerr coefficient $n_{2}$ with the strength of the TPA coefficient $\alpha_{2}$. This FOM is defined as FOM $=n_{2} /\left(\lambda \alpha_{2}\right)$. From our experiments we know that its value should be 0.5 or larger. Table 1 summarizes the results.

(a) Core Nonlinearity
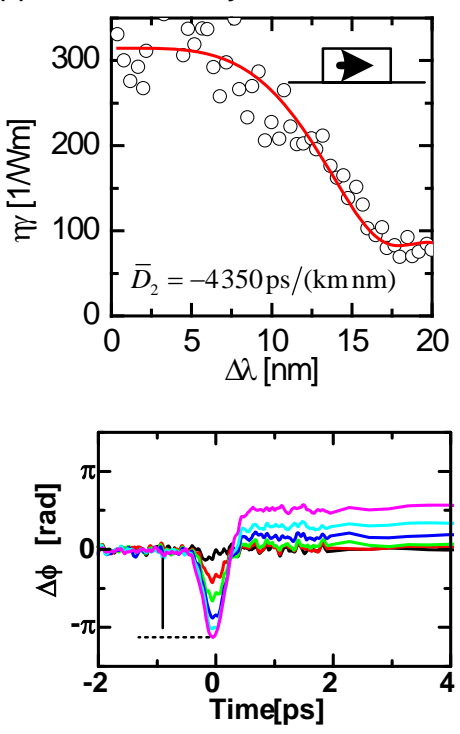

(b) Cladding Nonlinearity
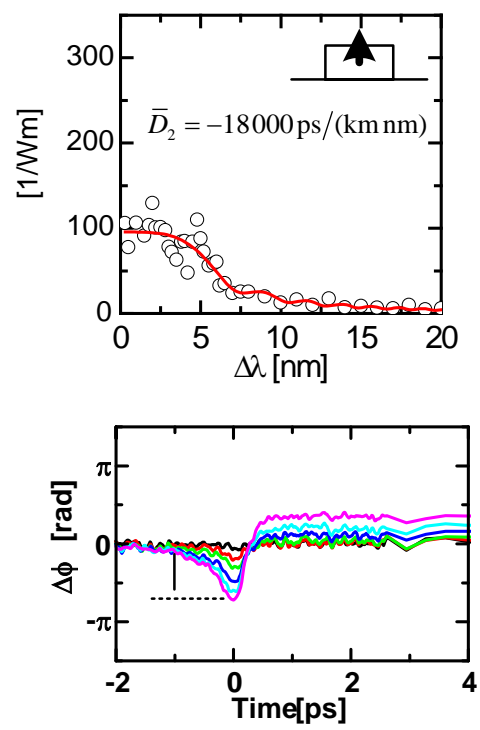

(c) Slot Nonlinearity
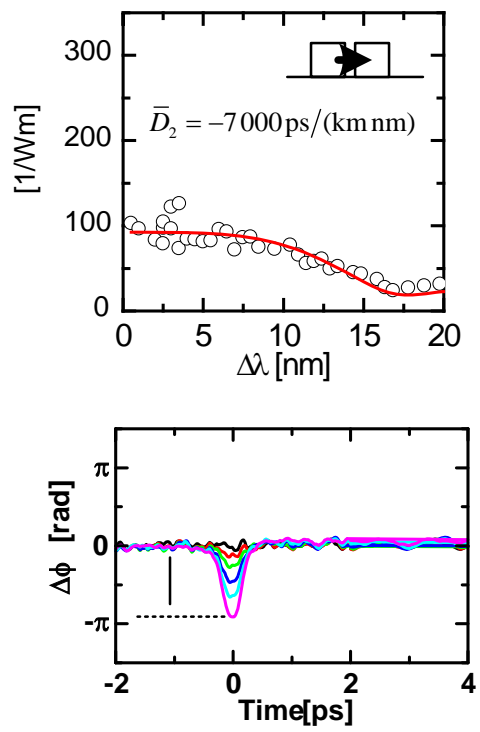

Fig. 2 (Upper row) Dependence of the nonlinearity parameter $\operatorname{Re}\{\gamma\}$ times $\eta$, the normalized four-wave mixing efficiency, as a function of the wavelength detuning $\Delta \lambda$; measurement $\left(^{\circ}\right)$ and fit $(-) . \eta$ describes the normalized degradation of the four-wave mixing efficiency with increasing phase mismatch, for perfect phase-matching $\eta=1$ holds. (Lower row) Pump light-induce phase change $\Delta \phi$ dynamics of the highly nonlinear waveguides for different pump power levels. Only the slot-nonlinear structure shows no TPA impeded slow phase dynamics.

Table 1: Summary of waveguide parameters of the three structures.

\begin{tabular}{|l|l|l|l|}
\hline Design & Core & Cladding & Slot \\
\hline$\gamma[1 /(\mathrm{W} \mathrm{km})]$ & 307000 & 108000 & 100000 \\
\hline FOM $_{\mathrm{TPA}}$ & 0.38 & 1.21 & 2.19 \\
\hline Height $[\mathrm{nm}]$ & 220 & 220 & 220 \\
\hline RibWidth $[\mathrm{nm}]$ & $360 \ldots 400$ & $360 \ldots 400$ & 220 \\
\hline SlotWidth $[\mathrm{nm}]$ & - & - & $160 \ldots 200$ \\
\hline
\end{tabular}




\section{IWC1.pdf}

\section{Nonlinear Signal Processing with Silicon Organic Hybrid Waveguide Structures}

To show the viability of the approach we give some examples of signal processing experiments performed with silicon organic hybrid waveguides. For instance, all-optical demultiplexing from $120 \mathrm{Gbit} / \mathrm{s}$ to $10 \mathrm{Gbit} / \mathrm{s}$ and $170 \mathrm{Gbit} / \mathrm{s}$ to $43 \mathrm{Gbit} / \mathrm{s}$ [7] has been shown in a slot-waveguide of $4 \mathrm{~mm}$ length. More recently, $40 \mathrm{Gbit} / \mathrm{s} \mathrm{RZ}$ and $56 \mathrm{Gbit} / \mathrm{s}$ DQPSK all-optical wavelength conversion based on the nonlinear FWM process as well as $43 \mathrm{Gbit} / \mathrm{s}$ all-optical wavelength conversion exploiting XPM nonlinearities have been demonstrated [8]-[10]. The scheme and the eye diagrams of the most recent all-optical wavelength conversion experiments are depicted in Fig. 3.

(a) Experimental Setup

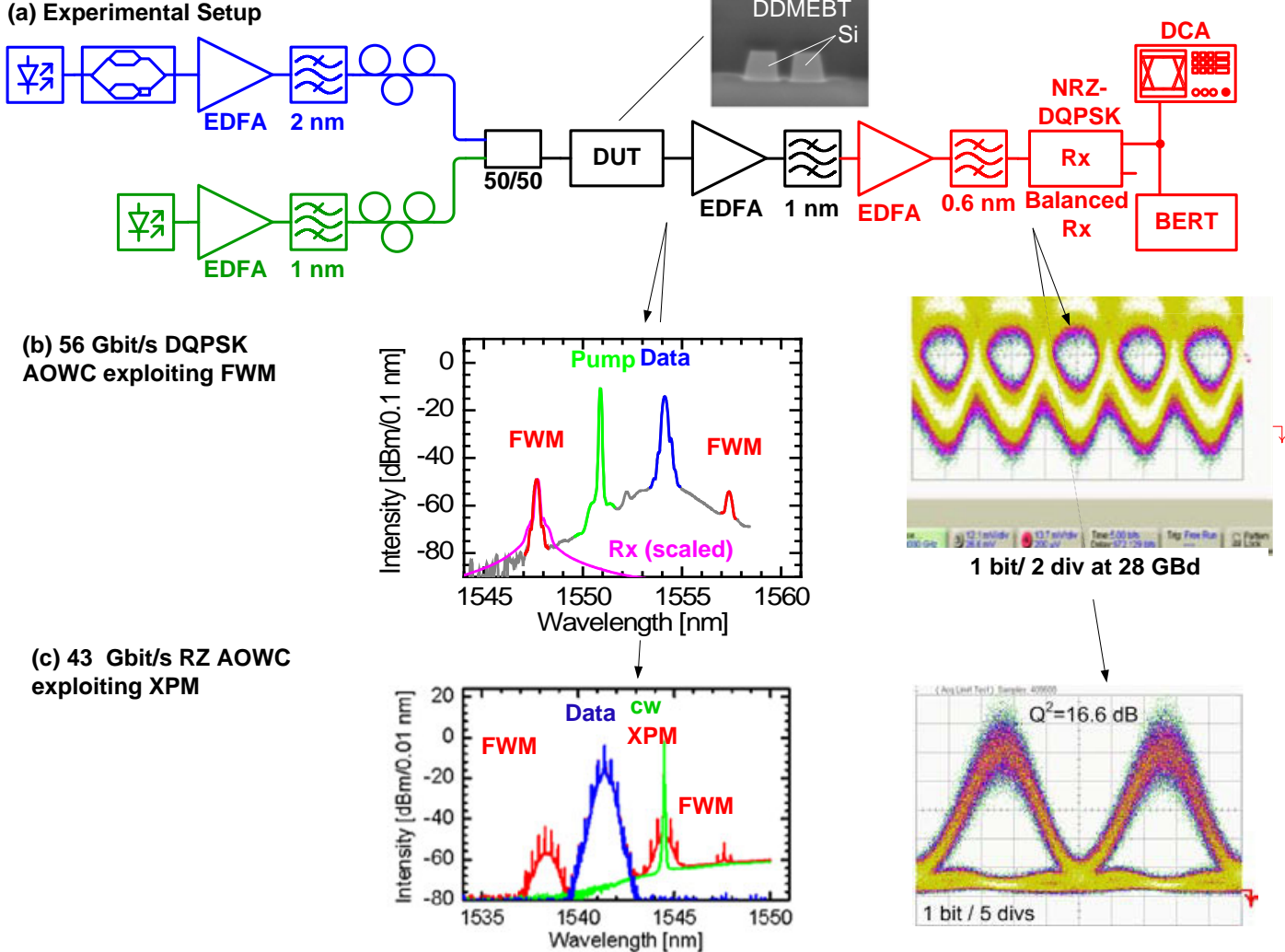

Fig. 3 All-optical wavelength conversion with the silicon-organic hybrid approach. (a) Experimental setup, (b) 56 Gbit/s all-optical DQPSK wavelength conversion exploiting the FWM nonlinear effect. (c) $43 \mathrm{Gbit} / \mathrm{s}$ all-optical RZ wavelength conversion exploiting the XPM nonlinear effect.

Acknowlegements: The authors acknowledge support from the Karlsruhe School of Optics \& Photonics (KSOP), the Center for Functional Nanostructures (CFN), the BMBF project MISTRAL and the EU project SOFI.

\section{References}

[1] C. Gunn, T.L Koch; "Silicon Photonics", in Optical fiber telecommunications V, edited by I.P. Kamniow, T. Li, A. Willner, AP 2008

[2] T. Baehr-Jones, M. Hochberg; " Silicon photonics: Slot machine". Nature Photonics 3, 193 - 194 (2009).

[3] B.G. Lee, K. Bergmann; Proc. OSA Annual Meeting, Rochester, paper FThS1, (2008).

[4] M. Lipson, "Guiding Modulating, and Emitting Light on Silicon-Challenges and Opportunities"; J. Lightw. Technol. 23, 4222 ff. (2005)

[5] J. Leuthold et al.; Proceedings of the IEEE 97, No. 7, pp. 1304-1316, ( 2009); doi: 10.1109/JPROC.2009.2016849

[6] C. Koos et al.; Optical Fiber Communnications Conference (OFC'08), Postdeadline Paper PDP25 (2008);

[7] C. Koos et al.; Nature Photon. 3, pp. 216-219, (2009); doi:10.1038/nphoton.2009.25

[8] T. Vallaitis et al.; Proc. Optical Fiber Communications Conf. 2009 (OFC'2009), San Diego, USA, Paper OWS3, ( 2009).

[9] T. Vallaitis et al.; Optical Fiber Communication Conference, Optical Society of America, pp. OTuN1 (2010).

[10] T. Vallaitis et al.; Intern. Conf. on Photonics in Switching (PS'09), Pisa, Italy, PDP3, Sept. 15-19, 2009; doi: 10.1109/PS.2009.5307823

[11] B. Esembeson, et al.; Adv. Mater. 19, 1-4, 2008; doi:10.1002/adma.200801552

[12] C. Koos, et. al.; Optics Express 15, No. 10, pp. 5976-5990, (2007). doi:10.1364/OE.15.005976

[13] T. Vallaitis, et al.; Optics Express, 17, No. 20, pp. 17357-17368, (2009). doi:10.1364/OE.17.017357

[14] R. Soref, et al.; IEEE J. Quantum Electron., QE-23, pp. 123-129, (1987)

[15] R. S. Jacobsen, et. al.; Nature 441, 199-202(11 May 2006)

[16] M. Hochberg et. al.; Optics Express, 15, no. 13, pp. 8401 ff., (2007)

[17] J.-M. Brosi et al.; Optics Express, 16, pp. 4177-4191, (2008). doi:10.1364/OE.16.004177

[18] T. Baehr-Jones et al.; Appl. Physics Letters, 92, 163303, (2008).

[19] L. Alloatti et al.; Proc. European Conf. on Optical Communications (ECOC'2010), Sept. 2010, accepted for publication. 